Service social

\title{
Clinical Social Work Practice with the Elderly, par Marion L. Beaver et Don Miller, Homewood (Ill.), Dorsey Press, 1985, 280 pages.
}

\section{Lise Darveau-Fournier}

Volume 34, numéro 1, 1985

Personnes âgées, milieux de vie et pratiques sociales

URI : https://id.erudit.org/iderudit/706261ar

DOI : https://doi.org/10.7202/706261ar

Aller au sommaire du numéro

Éditeur(s)

École de service social de l'Université Laval

ISSN

1708-1734 (numérique)

Découvrir la revue

Citer ce compte rendu

Darveau-Fournier, L. (1985). Compte rendu de [Clinical Social Work Practice with the Elderly, par Marion L. Beaver et Don Miller, Homewood (Ill.), Dorsey Press, 1985, 280 pages.] Service social, 34(1), 185-188.

https://doi.org/10.7202/706261ar d'utilisation que vous pouvez consulter en ligne.

https://apropos.erudit.org/fr/usagers/politique-dutilisation/ 
Pour terminer, les auteurs proposent au lecteur une grande aventure d'anticipation, des scénarios commentés, qui lui permettront non seulement d'examiner sa vie à travers celle des autres et de visualiser les situations et paysages qui seront les siens demain, mais aussi d'apercevoir les embûches qu'il voudra à tout prix éviter. Ces histoires-miroirs ont pour but de lui faire sentir aussi que les jeux ne sont pas faits, que chacun peut acquérir un certain pouvoir sur l'orientation de sa vie de retraite.

De lecture agréable, c'est un livre qu'on peut recommander non seulement aux responsables des cours de préparation à la retraite mais aussi à tous ceux qui souhaitent se faire une meilleure idée de ce qui les attend à cette étape de leur vie.

Suzanne ZAY

Clinical Social Work Practice with the Elderly, par Marion L. BeAver et Don MiLleR, Homewood (III.), Dorsey Press, 1985, 280 pages.

Ce volume traite de l'intervention sociale auprès des personnes âgées dans une perspective de prévention : primaire auprès des personnes en bonne santé, secondaire auprès de celles qui présentent des problèmes aigus et tertiaire auprès des non-autonomes. Le vieillissement est présenté dans une perspective de développement, comme une étape de vie unique qui a ses propres exigences et ses propres occasions de croissance.

Le volume se divise en huit chapitres. Les quatre premiers abordent surtout les principaux phénomènes liés au vieillissement, de même que les rôles principaux qui s'en dégagent pour le travailleur social ; les trois suivants traitent de l'intervention comme telle auprès de diverses catégories de personnes âgées qui vivent dans la communauté ou les institutions, alors que le dernier fait ressortir quelques-unes des difficultés de l'intervention préventive.

Dans le premier chapitre, les auteurs situent le vieillissement dans le cadre des étapes de vie formulées par Erikson; ils discutent particulièrement des nombreux changements biophysiologiques, psychologiques et sociaux que subissent les personnes âgées, qui entraînent des pertes de toutes sortes comme la diminution de l'acuité des sens et la perte de rôles, qui sont autant de sources de stress (pp. 10-30). À cet égard, on décrit les interventions du travailleur social comme suit: au niveau de la personne elle-même, viser à augmenter la compétence interactionnelle et l'autonomie, et affermir leur identité propre; au niveau de l'environnement, renforcer et supporter les réseaux sociaux informels, mettre les personnes âgées en relation avec les réseaux sociaux existants ou en train de se développer; fournir également des occasions d'action, de prise de décision et de maîtrise sur leur destinée, et restructurer certaines situations de manière à les rendre mieux adaptées aux personnes âgées (p. 9). 
Dans le deuxième chapitre, les auteurs indiquent que "l'objectif le plus important pour l'aidant professionnel est de s'efforcer de maintenir les personnes vieillissantes à des niveaux optimaux de fonctionnement" (p. 37). Ils constatent, par contre, que les travailleurs sociaux entrent souvent en contact avec les personnes âgées seulement après que celles-ci aient rencontré des difficultés dans leur transition à la vie de retraité. On préconise alors une intervention sociale préventive, tant primaire que secondaire et tertiaire, en indiquant que ces trois niveaux constituent un continuum et non des entités séparées.

L'intervention préventive primaire est définie comme celle qui tente de prévenir ou de contrer les problèmes avant qu'ils ne se produisent (p. 43). L'aide secondaire est présentée comme étant directe auprès des individus, familles et petits groupes, à partir de problèmes qu'ils rencontrent dans leur vie quotidienne, particulièrement ceux qui ont une maladie grave ou qui vivent une situation stressante (veuvage, retraite, etc.) (p. 66). Quant à l'intervention tertiaire, elle s'adresse surtout aux personnes moins autonomes en raison de divers handicaps; elle inclut la réhabilitation (p. 56). Une description des principaux rôles pour chaque niveau précité complète le chapitre: ceux de consultant-éducateur et d'avocat en prévention primaire, et ceux de clinicien, de courtier-avocat, de facilitateur et de "détecteur de besoins» (p. 69), en prévention secondaire et tertiaire. Les rôles d'enseignant, de mobilisateur, de planificateur et de distributeur de services (p. 70) peuvent être utiles à chacun des niveaux de prévention, ajoute-t-on.

Le troisième chapitre présente le service social clinique comme une pratique qui doit être éclectique (en termes de flexibilité conceptuelle et technique) à toutes les phases du processus d'intervention. Les procédures de traitement y sont décrites selon cinq approches : psychosociale, centrée sur le client, intervention en situation de crise, behaviorale, cognitive. Quant au quatrième chapitre, il porte sur la santé et le bien-être des personnes âgées bien portantes, lesquelles sont décrites comme des individus qui peuvent avoir expérimenté ou non des pertes importantes au plan social, économique ou psychologique, mais qui ont trouvé des moyens de s'adapter à ces pertes sans trop de difficulté. Les auteurs traitent de qualité de vie et du rôle que peut jouer le travailleur social à cet effet, en le situant dans une optique d'intervention préventive primaire. On y cite, entre autres, en faisant ressortir les avantages spécifiques de chacun, quatre exemples d'intervention préventive auprès des bien-portants : les programmes de formation, les réseaux de voisinage, le travail de groupe avec les familles des personnes âgées et les groupes naturels d'entraide.

Le cinquième chapitre concerne l'intervention auprès des personnes âgées qui rencontrent des problèmes aigus de fonctionnement. On y traite, entre autres, des difficultés reliées au stress qu'occasionnent souvent la relocalisation, l'isolement social, le fonctionnement sexuel, les relations conjugales et familiales, le veuvage et la retraite. On y discute aussi de santé mentale, de névrose du vieillissement, de réaction au chagrin, de dépression et d'alcoolisme. Selon les auteurs, l'intervenant doit aider la personne âgée à demeurer le plus 
possible maître de sa situation, à prendre elle-même les décisions qui la concernent, à faire ses propres choix; il doit aussi l'aider à développer de nouvelles interactions sociales et de nouvelles amitiés. Ce type d'intervention se situe dans une perspective de prévention secondaire.

Le sixième chapitre aborde l'intervention préventive tertiaire avec les personnes âgées qui continuent de vivre dans la communauté, mais qui ne sont plus autonomes en raison de carences physiques, mentales ou sociales, importantes et chroniques, et qui ont besoin d'aide dans une ou plusieurs activités de leur vie quotidienne (p. 172). Les principaux problèmes qui affectent les personnes âgées y sont décrits, incluant la maladie d'Alzheimer. On y retrouve aussi une notion élargie des soins prolongés hors-institution, lesquels "comprennent non seulement un effort de réhabilitation thérapeutique pour améliorer les niveaux de fonctionnement, mais aussi des efforts qui visent à maintenir les niveaux actuels de fonctionnement, à ralentir la détérioration et à assurer des soins de protection durant l'étape terminale de vie». (P. 177.) On suggère, enfin, en vue d'augmenter la qualité de vie d'offrir une gamme de services au domicile même de la personne-cliente (aux plans médical, social, nutritionnel, ménager, etc.), complétés par d'autres plus complexes (cliniques médicales et psychiatriques, counseling individuel et de groupe, assistance légale, etc.) disponibles dans les organismes appropriés (p. 177).

Les auteurs rappellent que l'intervention préventive doit aussi rejoindre ceux qui prennent soin des personnes âgées à domicile (conjoint, enfants, parents, amis ou autres) afin de les aider à comprendre les maladies de la personne dont ils ont charge, à acquérir les connaissances et les habiletés requises pour remplir efficacement leur rôle et à entrer en contact avec les ressources qui peuvent leur être utiles. On mentionne, à cet effet, des programmes de groupe à des fins d'éducation, de support ou de traitement, destinés aux bien-portants qui prennent soin de personnes non autonomes (p. 183).

Le chapitre suivant aborde le sujet des personnes âgées en institution, dans une perspective d'intervention préventive tertiaire. L'hôpital de soins prolongés doit "offrir un environnement quotidien qui stimule et renforce des comportements cognitifs et sociaux appropriés", précise-t-on (p. 194). On suggère que l'intervention de groupe puisse être utilisée pour aider les patients à se socialiser et à acquérir les habiletés de base nécessaires à la vie quotidienne. Et les auteurs insistent sur l'importance d'aider les bénéficiaires à s'impliquer dans le plus grand nombre possible de rôles significatifs; ils suggèrent donc différents programmes susceptibles d'améliorer le fonctionnement psychosocial en institution, entre autres: la thérapie de milieu, l'orientation dans la réalité, divers types de groupes (de remotivation, de thérapie par la musique et par l'art, de réminiscence) ainsi que des comités de bénéficiaires. On trouve aussi, dans ce chapitre, plusieurs références sur divers types de groupes qui peuvent être utilisés avec des personnes âgées. Le rôle du travailleur social y est considéré particulièrement dans le sens de promouvoir le meilleur fonctionnement possible des personnes et d'augmenter la qualité de vie dans le milieu. Cela implique un travail de collaboration avec tous les autres intervenants concernés 
(personnel de soin, bénévoles, etc.) ainsi qu'avec les réseaux sociaux informels et les ressources formelles de la communauté. On y rappelle que l'intervention sociale auprès de la personne âgée et de la famille doit commencer tôt, c'està-dire dès le moment où on effectue des démarches en vue d'un placement.

Le dernier chapitre présente les principales difficultés de l'intervention sociale, qui proviendraient de l'état des connaissances sur le vieillissement et sur l'intervention sociale préventive, des politiques gouvernementales, des procédures des organismes, et des praticiens eux-mêmes. Les auteurs insistent toutefois pour dire que ces difficultés ne sont pas insurmontables mais qu'il est important de les connaître pour les prévenir ou les contourner. Ce chapitre contient aussi des indications sur les attitudes que peuvent prendre les travailleurs sociaux et sur les rôles qu'ils peuvent jouer afin de provoquer des changements et d'améliorer la qualité de l'intervention psychosociale auprès des personnes âgées.

Les auteurs précisent que ce volume s'adresse aux professeurs et aux étudiants impliqués dans des programmes de formation ainsi qu'aux intervenants qui travaillent directement auprès des personnes âgées. Ils ajoutent que ce livre peut également être utile aux administrateurs, planificateurs et superviseurs qui souhaitent augmenter leurs connaissances et leurs habiletés en intervention préventive. Les professionnels des sciences infirmières, de la médecine et de la psychiatrie peuvent également en tirer profit.

Dans l'ensemble, les sujets sont traités avec clarté. Le fait que chaque chapitre soit abondamment illustré d'exemples concrets contribue à l'originalité et à l'intérêt du volume, en plus d'en faciliter la compréhension; il s'agit habituellement d'histoires de cas et de plans d'intervention, souvent même accompagnés de questions adressées au lecteur, de nature à guider la réflexion ou la discussion.

Les auteurs s'attardent davantage aux aspects cliniques de l'intervention ; on retrouve toutefois, à diverses reprises, des suggestions d'intervention de type communautaire. II m'apparait important que cette dimension soit davantage mise en lumière, car le travail avec la communauté, avec ses réseaux formels et informels, me semble indispensable au succès de toute intervention préventive, qu'elle soit primaire, secondaire ou tertiaire.

École de service social,

Lise Darveau-Fournier

Université Laval.

La retraite au féminin, par Philippe PITAUD, Paris, Pierre Horay, 1983, 221 pages.

Jusqu'à très récemment, la retraite était perçue comme un phénomène masculin, même si le travail de la femme hors du foyer n'est pas réellement quelque chose de nouveau. Pendant longtemps c'est donc dans le cadre du passage de l'homme à la retraite qu'on a examiné cette condition. 